\title{
Boundary value problem for a class of fractional integro-differential coupled systems with Hadamard fractional calculus and impulses
}

\author{
Kaihong Zhao ${ }^{1 *}$, Leping Suo ${ }^{1}$ and Yongzhi Liao²
}

\author{
"Correspondence: \\ zhaokaihongs@126.com \\ 'Department of Applied \\ Mathematics, Kunming University of \\ Science and Technology, Kunming, \\ China \\ Full list of author information is \\ available at the end of the article
}

\begin{abstract}
This paper considers the boundary value problem for a class of fractional integro-differential coupled systems with Hadamard fractional calculus and impulses. Some sufficient conditions of the existence and uniqueness are obtained by means of the Banach contraction principle and Leray-Schauder alternative. We also give some interesting examples to illustrate the effectiveness of our main results.
\end{abstract}

MSC: 34B10; 34B15; 34B37

Keywords: Boundary value problem; Hadamard fractional differential coupled system; Impulses; Leray-Schauder alternative theorem; Existence and uniqueness

\section{Introduction}

In 1695, L'Hôpital asked what was $\frac{d^{n} y}{d x^{n}}$ if $n=\frac{1}{2}$ in his letter to Leibniz. This year is generally regarded as the birthday of fractional calculus. Hereafter, Leibniz, J. Bernoulli, Euler, Lagrange, Laplace, Lacroix, Fourier, Abel, Cantor, De Morgen, Ya Sonin, Riemann, Liouville, Caputo, et al. have made important contributions to the definition of fractional calculus. In 1830s, Riemann and Liouville defined the integral and derivative which is now called Riemann-Liouville (R-L) fractional calculus by the Cauchy integral formula. Subsequently, many famous and important fractional integrals and derivatives have been proposed, for example, Grünwald-Letnikov fractional derivative, Caputo fractional derivative, Weyl fractional calculus, Hadamard fractional calculus, and so on. As for the history of fractional calculus, the readers can refer to the literature $[1,2]$.

The fractional-order calculus as a good tool is used to establish the mathematical model describing many actual phenomena and processes. For example, the fractional differential equations can describe the diffusion processes (see $[3,4]$ ), the mechanical properties of materials (see [5-8]), the signal processing (see [9]), the image processing (see [10]), the behavior of viscoelastic and visco-plastic materials under external influences (see [11, 12]), the pharmacokinetics (see [13-15]), the bioengineering (see $[16,17])$, the control theory (see $[18,19])$, and so on. In addition there are some applications of fractional calculus within various fields of mathematics itself, e.g., in the analytical investigation of various

(c) The Author(s) 2019. This article is distributed under the terms of the Creative Commons Attribution 4.0 International License (http://creativecommons.org/licenses/by/4.0/), which permits unrestricted use, distribution, and reproduction in any medium, provided you give appropriate credit to the original author(s) and the source, provide a link to the Creative Commons license, and indicate if changes were made. 
types of special functions (see [20]). Therefore, the fractional differential equation has been widely focused and studied in depth. There have been some monographs and textbooks for the readers to learn use fractional calculus theories and methods (see [2, 21-25]). In the last few decades, there have been many papers dealing with fractional differential equation involving Riemann-Liouville and Caputo fractional derivatives (see [26-43]). In fact, the Hadamard fractional derivative is one of the most famous fractional calculi which was put forward by Hadamard in 1892. This type of fractional derivative differs from other types of derivatives. Its main feature is that the integral kernel contains a logarithmic function of arbitrary exponent in definition. Recently, there have been several papers dealing with Hadamard fractional differential equation (see [44-67]). However, these papers rarely considered the Hadamard fractional differential coupled equations. Therefore, it is interesting and challenging to study the Hadamard nonlinear fractional differential coupled system with impulses. So, in this paper we mainly study the following impulsive fractional differential coupled system with Hadamard fractional calculus:

$$
\left\{\begin{array}{l}
\mathrm{RLH}_{t_{k}}^{\alpha}\left[u(t)-{ }_{H} J_{t_{k}}^{\alpha} e(t, u(t), v(t))\right]=g(t, u(t), v(t)), \quad t \in J=[a, T], t \neq t_{k}, \\
\operatorname{RLH}_{t_{k}} D_{t_{k}}^{\beta}\left[v(t)-{ }_{H} J_{t_{k}}^{\beta} f(t, u(t), v(t))\right]=h(t, u(t), v(t)), \quad t \in J=[a, T], t \neq t_{k}, \\
\operatorname{RLH} D_{t_{k}}^{\alpha-1} u\left(t_{k}^{+}\right)-\mathrm{RLH}_{t_{k}}^{\alpha-1} u\left(t_{k}^{-}\right)=I_{k}\left(u\left(t_{k}\right)\right), \quad k=1,2, \ldots, m, \\
\operatorname{RLH}_{t_{k}}^{\beta-1} v\left(t_{k}^{+}\right)-\mathrm{RLH}_{t_{k}}^{\beta-1} v\left(t_{k}^{-}\right)=J_{k}\left(v\left(t_{k}\right)\right), \quad k=1,2, \ldots, m, \\
c \cdot \operatorname{RLH}_{a}^{\alpha-1} u(a)=u(T), \quad d \cdot \operatorname{RLH} D_{a}^{\beta-1} v(a)=v(T),
\end{array}\right.
$$

where $a>0,1<\alpha, \beta<2, c, d \in \mathbb{R}, I_{k}, J_{k} \in C(\mathbb{R}, \mathbb{R}) .{ }_{\mathrm{RLH}} D_{t_{k}}^{\alpha}$, RLH $D_{t_{k}}^{\beta}$ denote the left-sided Riemann-Liouville type Hadamard fractional derivatives of order $\alpha$ and $\beta{ }_{H} J_{t_{k}}^{\alpha},{ }_{H} J_{t_{k}}^{\beta}$ denote the left-sided Hadamard fractional integrals of order $\alpha$ and $\beta . e, f, g, h: J \times \mathbb{R}^{2} \rightarrow \mathbb{R}$ are some given continuous functions and impulsive points, $\left\{t_{k}\right\}_{k=1}^{m}$ satisfies $a=t_{0}<t_{1}<t_{2}<$ $\cdots<t_{m}<t_{m+1}=T$. RLH $D_{t_{k}}^{\alpha-1} u\left(t_{k}^{+}\right), \mathrm{RLH}_{t_{k}} D^{\alpha-1} u\left(t_{k}^{-}\right),{ }_{\mathrm{RLH}} D_{t_{k}}^{\beta-1} v\left(t_{k}^{+}\right)$, and $\mathrm{RLH}_{t_{k}} D^{\beta-1} v\left(t_{k}^{-}\right)$represent the right and left limits and satisfy the left continuity at $t=t_{k}, k=1,2, \ldots, m$.

In addition, some other inspiration for studying system (1.1) comes from the literature [48, 49]. In [48], the authors considered the existence and finite-time stability results of Hadamard type impulsive fractional differential equations as follows:

$$
\left\{\begin{array}{l}
{ }_{H} D_{1}^{\alpha} u(t)=f\left(t, u(t), \max _{\xi \in[\beta t, t]} u(\xi)\right), \quad \alpha \in(0,1), t \in[1, e] \backslash \Theta, \beta \in(0,1), \\
{ }_{H} J_{1}^{1-\alpha} u\left(t_{i}^{+}\right)-{ }_{H} J_{1}^{1-\alpha} u\left(t_{i}^{-}\right)=a_{i} u\left(t_{i}^{-}\right)+b_{i}, \quad a_{i}, b_{i}>0, i=1,2, \ldots, m, \\
{ }_{H} J_{1}^{1-\alpha} u(1)=u_{0}, \quad u_{0}>0
\end{array}\right.
$$

with initial condition $u(t)=\phi(t), t \in[\beta, 1]$, where ${ }_{H} D_{1}^{\alpha}$ denotes the left-sided RiemannLiouville type Hadamard fractional derivative of order $\alpha,{ }_{H} J_{1}^{1-\alpha}$ denotes the left-sided Hadamard fractional integral of order $1-\alpha$, and $\Theta=\left\{t_{1}, t_{2}, \ldots, t_{m}\right\}$ satisfying $1=t_{0}<$ $t_{1}<\cdots<t_{m}<t_{m+1}=e . f: J \times \mathbb{R} \times \mathbb{R} \rightarrow \mathbb{R}$ is a Carathéodory function, and $u\left(t_{i}^{+}\right)=$ $\lim _{\epsilon \rightarrow 0^{+}} u\left(t_{i}+\epsilon\right), u\left(t_{i}^{-}\right)=\lim _{\epsilon \rightarrow 0^{-}} u\left(t_{i}+\epsilon\right)$.

In [49], the author discussed the existence and uniqueness results of solutions for the Hadamard and Riemann-Liouville fractional neutral functional integro-differential equa- 
tions with finite delay described by

$$
\left\{\begin{array}{l}
{ }_{H} D^{\alpha}\left[u(t)-\sum_{i=1}^{m} I^{\beta_{i}} h_{i}\left(t, u_{t}\right)\right]=f\left(t, u_{t}\right), \quad t \in J=[1, T] \\
u(t)=\varphi(t), \quad t \in[1-r, 1], r>0
\end{array}\right.
$$

where ${ }_{H} D^{\alpha}$ denotes the left-sided Riemann-Liouville type Hadamard fractional derivative of order $\alpha, 0<\alpha \leq 1, I^{\beta_{i}}$ is the Riemann-Liouville fractional integral of order $\beta_{i}>0, i=$ $1,2, \ldots, m, f, h_{i}: J \times C([-r, 0], \mathbb{R}) \rightarrow \mathbb{R}$ are given continuous functions, $\varphi \in C([1-r, 1], \mathbb{R})$ with $\varphi(1)=0$. For any function $u$ defined on $[1-r, T]$ and any $t \in J, u_{t}(\theta)=u(t+\theta)$, $\theta \in[-r, 0]$ denotes the element of $C([-r, 0], \mathbb{R})$. The author derived the existence of solutions by the Leray-Schauder alternative and established the uniqueness of solutions by the Banach contraction principle.

The rest of this paper is organized as follows. In Sect. 2, we recall some useful preliminaries. In Sect. 3, we shall prove the existence and uniqueness of solutions for system (1.1). In Sect. 4, some examples are also provided to illustrate the effectiveness of our main results. Finally, the conclusion is given to simply recall our studied contents and obtained results in Sect. 5.

\section{Preliminaries}

In this section, we introduce some notations and definitions of Hadamard fractional calculus and present preliminary results needed in our proofs later.

Definition 2.1 ([22]) For $a \geq 0$, the left-sided Hadamard fractional integral of order $\alpha>0$ for a function $u:(a, \infty) \rightarrow \mathbb{R}$ is defined as

$$
{ }_{H} J_{a}^{\alpha} u(t)=\frac{1}{\Gamma(\alpha)} \int_{a}^{t}\left(\ln \frac{t}{s}\right)^{\alpha-1} u(s) \frac{d s}{s}
$$

where $\Gamma(\cdot)$ is the gamma function.

Definition 2.2 ([22]) For $a \geq 0$, the left-sided Riemann-Liouville type Hadamard fractional derivative of order $\alpha>0$ for a function $u:(a, \infty) \rightarrow \mathbb{R}$ is defined by

$$
\mathrm{RLH} D_{a}^{\alpha} u(t)=\frac{1}{\Gamma(n-\alpha)}\left(t \frac{d}{d t}\right)^{n} \int_{a}^{t}\left(\ln \frac{t}{s}\right)^{n-\alpha+1} u(s) \frac{d s}{s}, \quad n-1<\alpha<n, n=[\alpha]+1,
$$

where $[\alpha]$ denotes the integer part of the real number $\alpha>0$, and $\Gamma(\cdot)$ is the gamma function.

Lemma 2.1 ([22]) For $a>0$, assume that $u \in C(a, T) \cap L^{1}(a, T)$ with a left-sided RiemannLiouville type Hadamard fractional derivative of order $\alpha>0$. Then

$$
{ }_{H} J_{a \mathrm{RLH}}^{\alpha} D_{a}^{\alpha} u(t)=u(t)+c_{1}\left(\ln \frac{t}{a}\right)^{\alpha-1}+c_{2}\left(\ln \frac{t}{a}\right)^{\alpha-2}+\cdots+c_{n}\left(\ln \frac{t}{a}\right)^{\alpha-n}
$$

for some $c_{i} \in \mathbb{R}, i=1,2, \ldots, n-1, n=[\alpha]+1$. 
Lemma 2.2 ([44]) Let $\alpha>0, \beta>0$, and $0<a<\infty$. Then the following properties hold:

$$
\begin{aligned}
& \mathrm{RLH}_{a}^{\alpha}\left(\ln \frac{t}{a}\right)^{\beta-1}(x)=\frac{\Gamma(\beta)}{\Gamma(\beta-\alpha)}\left(\ln \frac{x}{a}\right)^{\beta-\alpha-1}, \\
& J_{a}^{\alpha}\left(\ln \frac{t}{a}\right)^{\beta-1}(x)=\frac{\Gamma(\beta)}{\Gamma(\beta+\alpha)}\left(\ln \frac{x}{a}\right)^{\beta+\alpha-1}, \\
& \mathrm{RLH}_{a}^{\alpha} D_{a}^{\alpha} J_{a}^{\alpha} u(t)=u(t)
\end{aligned}
$$

Lemma 2.3 ([68]) If $E$ is a real Banach space and $F: E \rightarrow E$ is a contraction mapping, then $F$ has a unique fixed point in $E$.

Lemma 2.4 (Leray-Schauder alternative theorem [61]) Let $U$ be a normed linear space and $F: U \rightarrow U$ be a completely continuous operator (i.e., a map that restricted to any bounded set in $U$ is compact). Let

$$
\varepsilon(F)=\{x \in U: x=k F(x), 0<k<1\}
$$

then either the set $\varepsilon(F)$ is unbounded, or $F$ has at least one fixed point.

For the convenient statements, we introduce the notation as follows: $t_{0}=a, t_{m+1}=T$, $I_{0}(t)=J_{0}(t) \equiv 0$. Let $C[a, T]$ be the Banach space of all continuous functions from $[a, T] \rightarrow$ $\mathbb{R}$ with the norm $\|\omega\|=\sup _{t \in[a, T]}|\omega(t)|$. For $1<\gamma<2$, we define

$$
\begin{aligned}
\operatorname{PC}_{\gamma}[a, T]= & \left\{\omega: \omega(t) \in C\left(t_{k}, t_{k+1}\right],{ }_{\mathrm{RLH}} D_{t_{k}}^{\gamma-1} \omega\left(t_{k}^{-}\right), \mathrm{RLH}_{t_{k}}^{\gamma-1} \omega\left(t_{k}^{+}\right)\right. \text {all exist } \\
& \text { and satify } \left.\mathrm{RLH}_{t_{k}}^{\gamma-1} \omega\left(t_{k}^{-}\right)=\mathrm{RLH}_{\mathrm{RL}_{k}}^{\gamma-1} \omega\left(t_{k}\right), k=0,1,2, \ldots, m\right\} .
\end{aligned}
$$

Obviously, $\mathrm{PC}_{\gamma}[a, T]$ is a Banach space equipped with the norm $\|\omega\|_{\mathrm{PC}_{\gamma}}=\|\omega(t)\|_{C}$. The space $X=\mathrm{PC}_{\alpha}[a, T] \times \mathrm{PC}_{\beta}[a, T]$ equipped with the norm $\|(u, v)\|=\max \left\{\|u\|_{\mathrm{PC}_{\alpha}},\|v\|_{\mathrm{PC}_{\beta}}\right\}$ is also a Banach space.

Definition 2.3 A pair of functions $(u(t), v(t)) \in X=\mathrm{PC}_{\alpha}[a, T] \times \mathrm{PC}_{\beta}[a, T]$ is called to be a solution of $(1.1)$ if $(u(t), v(t))$ satisfy all the equations and boundary value conditions of system (1.1).

Lemma 2.5 Assume that the functions e, $g \in C[a, T])$ and $I_{k} \in C(\mathbb{R}, \mathbb{R})$. If $\delta \triangleq c \Gamma(\alpha)-$ $\left(\ln \frac{T}{t_{m}}\right)^{\alpha-1} \neq 0$, then, for given $v(t) \in \mathrm{PC}_{\beta}[a, T]$, a function $u(t) \in \mathrm{PC}_{\alpha}[a, T]$ is a solution of the impulsive Hadamard fractional differential equation

$$
\left\{\begin{array}{l}
\operatorname{RLH}_{t_{k}}^{\alpha}\left[u(t)-{ }_{H} J_{t_{k}}^{\alpha} e(t, u(t), v(t))\right]=g(t, u(t), v(t)), \quad 1<\alpha<2, \\
{ }_{\mathrm{RLH}} D_{t_{k}}^{\alpha-1} u\left(t_{k}^{+}\right)-\mathrm{RLH}_{t_{k}}^{\alpha-1} u\left(t_{k}^{-}\right)=I_{k}\left(u\left(t_{k}\right)\right), \quad k=1,2, \ldots, m, \\
c \cdot \operatorname{RLH} D_{a}^{\alpha-1} u(a)=u(T),
\end{array}\right.
$$


if and only if $u(t) \in C[a, T] \cap \mathrm{PC}_{\alpha}[a, T]$ is a solution of the integral equation

$$
\begin{aligned}
u(t)= & { }_{H} J_{t_{k}}^{\alpha} g(t, u(t), v(t))+{ }_{H} J_{t_{k}}^{\alpha} e(t, u(t), v(t))+c^{*}\left(\ln \frac{t}{t_{k}}\right)^{\alpha-1}+\frac{\Lambda}{\Gamma(\alpha)} \sum_{i=1}^{k}\left[I_{i}\left(u\left(t_{i}\right)\right)\right. \\
& \left.+{ }_{H} J_{t_{i-1}}^{1} g\left(t_{i}, u\left(t_{i}\right), v\left(t_{i}\right)\right)+{ }_{H} J_{t_{i-1}}^{1} e\left(t_{i}, u\left(t_{i}\right), v\left(t_{i}\right)\right)\right]\left(\ln \frac{t}{t_{k}}\right)^{\alpha-1}, \quad t \in\left(t_{k}, t_{k+1}\right],
\end{aligned}
$$

where $k=0,1,2, \ldots, m, \Lambda=\left\{\begin{array}{l}0, t \in\left[a, t_{1}\right], \\ 1, t \in\left(t_{1}, T\right],\end{array}\right.$, and

$$
\begin{aligned}
c^{*}= & \frac{1}{\delta}\left({ }_{H} J_{t_{m}}^{\alpha} g(T, u(T), v(T))+{ }_{H} J_{t_{m}}^{\alpha} e(T, u(T), v(T))+\frac{\left(\ln \frac{T}{t_{m}}\right)^{\alpha-1}}{\Gamma(\alpha)} \sum_{i=1}^{m}\left[I_{i}\left(u\left(t_{i}\right)\right)\right.\right. \\
& \left.\left.+{ }_{H} J_{t_{i-1}}^{1} g\left(t_{i}, u\left(t_{i}\right), v\left(t_{i}\right)\right)+{ }_{H} J_{t_{i-1}}^{1} e\left(t_{i}, u\left(t_{i}\right), v\left(t_{i}\right)\right)\right]\right) .
\end{aligned}
$$

Proof When $t \in\left[a, t_{1}\right]=\left[t_{0}, t_{1}\right]$, applying the Hadamard fractional integral operator on both sides of the first equation in (2.1), that is,

$$
{ }_{H} J_{t_{0} \mathrm{RLH}}^{\alpha} D_{t_{0}}^{\alpha}\left[u(t)-{ }_{H} J_{t_{0}}^{\alpha} e(t, u(t), v(t))\right]={ }_{H} J_{t_{0}}^{\alpha} g(t, u(t), v(t)),
$$

we have

$$
u(t)={ }_{H} J_{t_{0}}^{\alpha} g(t, u(t), v(t))+{ }_{H} J_{t_{0}}^{\alpha} e(t, u(t), v(t))+c_{1}\left(\ln \frac{t}{t_{0}}\right)^{\alpha-1}+d_{1}\left(\ln \frac{t}{t_{0}}\right)^{\alpha-2}
$$

where $c_{1}$ and $d_{1}$ are some constants. In the light of the existence of $u(a)$, we have $d_{1}=0$.

In view of Lemmas 2.1-2.2, we obtain

$$
\begin{aligned}
\operatorname{RLH} D_{t_{0}}^{\alpha-1} u(t)= & { }_{\mathrm{RLH}} D_{t_{0}}^{\alpha-1} J_{t_{0}}^{\alpha} g(t, u(t), v(t))+{ }_{\mathrm{RLH}} D_{t_{0}}^{\alpha-1} J_{t_{0}}^{\alpha} e(t, u(t), v(t)) \\
& +c_{1 H} D_{t_{0}}^{\alpha-1}\left(\ln \frac{t}{t_{0}}\right)^{\alpha-1} \\
= & { }_{H} J_{t_{0}}^{1} g(t, u(t), v(t))+{ }_{H} J_{t_{0}}^{1} e(t, u(t), v(t))+c_{1} \Gamma(\alpha) .
\end{aligned}
$$

(2.4) gives that

$$
\operatorname{RLH}_{t_{0}}^{\alpha-1} u\left(t_{0}\right)=c_{1} \Gamma(\alpha) .
$$

According to (2.3) and (2.4), we get

$$
\mathrm{RLH}_{t_{0}}^{\alpha-1} u\left(t_{1}^{-}\right)={ }_{H} J_{t_{0}}^{1} g\left(t_{1}, u\left(t_{1}\right), v\left(t_{1}\right)\right)+{ }_{H} J_{t_{0}}^{1} e\left(t_{1}, u\left(t_{1}\right), v\left(t_{1}\right)\right)+c_{1} \Gamma(\alpha)
$$

and

$$
u(t)={ }_{H} J_{t_{0}}^{\alpha} g(t, u(t), v(t))+{ }_{H} J_{t_{0}}^{\alpha} e(t, u(t), v(t))+c_{1}\left(\ln \frac{t}{t_{0}}\right)^{\alpha-1}, \quad t \in\left[t_{0}, t_{1}\right] .
$$


When $t \in\left(t_{1}, t_{2}\right]$, there are similar to have

$$
u(t)={ }_{H} J_{t_{1}}^{\alpha} g(t, u(t), v(t))+{ }_{H} J_{t_{1}}^{\alpha} e(t, u(t), v(t))+c_{2}\left(\ln \frac{t}{t_{1}}\right)^{\alpha-1}+d_{2}\left(\ln \frac{t}{t_{1}}\right)^{\alpha-2},
$$

where $c_{2}$ and $d_{2}$ are some constants. In the light of the existence of ${ }_{\mathrm{RLH}} D_{t_{1}}^{\alpha-1} u\left(t_{1}^{+}\right)$, we have $d_{2}=0$, and

$$
\mathrm{RLH}_{t_{1}}^{\alpha-1} u\left(t_{1}^{+}\right)=c_{2} \Gamma(\alpha)
$$

It follows from (2.6), (2.9), and the second equation of (2.1) that

$$
c_{2}-c_{1}=\frac{1}{\Gamma(\alpha)}\left[I_{1}\left(u\left(t_{1}\right)\right)+{ }_{H} J_{t_{0}}^{1} g\left(t_{1}, u\left(t_{1}\right), v\left(t_{1}\right)\right)+{ }_{H} J_{t_{0}}^{1} e\left(t_{1}, u\left(t_{1}\right), v\left(t_{1}\right)\right)\right]
$$

and

$$
u(t)={ }_{H} J_{t_{1}}^{\alpha} g(t, u(t), v(t))+{ }_{H} J_{t_{1}}^{\alpha} e(t, u(t), v(t))+c_{2}\left(\ln \frac{t}{t_{1}}\right)^{\alpha-1}, \quad t \in\left(t_{1}, t_{2}\right] .
$$

Repeating the above calculation process, for $t \in\left(t_{k}, t_{k+1}\right], k=1,2, \ldots, m$, we obtain

$$
c_{k+1}-c_{k}=\frac{1}{\Gamma(\alpha)}\left[I_{k}\left(u\left(t_{k}\right)\right)+{ }_{H} J_{t_{k-1}}^{1} g\left(t_{k}, u\left(t_{k}\right), v\left(t_{k}\right)\right)+{ }_{H} J_{t_{k-1}}^{1} e\left(t_{k}, u\left(t_{k}\right), v\left(t_{k}\right)\right)\right]
$$

and

$$
u(t)={ }_{H} J_{t_{k}}^{\alpha} g(t, u(t), v(t))+{ }_{H} J_{t_{k}}^{\alpha} e(t, u(t), v(t))+c_{k+1}\left(\ln \frac{t}{t_{k}}\right)^{\alpha-1}, \quad t \in\left(t_{k}, t_{k+1}\right] .
$$

From (2.12) and (2.13), we have

$$
\begin{aligned}
c_{m+1}-c_{1}= & \frac{1}{\Gamma(\alpha)} \sum_{k=1}^{m}\left[I_{k}\left(u\left(t_{k}\right)\right)+{ }_{H} J_{t_{k-1}}^{1} g\left(t_{k}, u\left(t_{k}\right), v\left(t_{k}\right)\right)\right. \\
& \left.+{ }_{H} J_{t_{k-1}}^{1} e\left(t_{k}, u\left(t_{k}\right), v\left(t_{k}\right)\right)\right]
\end{aligned}
$$

and

$$
\begin{aligned}
u(T)=u\left(t_{m+1}\right)= & { }_{H} J_{t_{m}}^{\alpha} g(T, u(T), v(T))+{ }_{H} J_{t_{m}}^{\alpha} e(T, u(T), v(T)) \\
& +c_{m+1}\left(\ln \frac{T}{t_{m}}\right)^{\alpha-1} .
\end{aligned}
$$

In the light of (2.5), (2.14), (2.15), and the third equation of (2.1), we have

$$
\begin{aligned}
c_{1}= & \frac{1}{\delta}\left({ }_{H} J_{t_{m}}^{\alpha} g(T, u(T), v(T))+{ }_{H} J_{t_{m}}^{\alpha} e(T, u(T), v(T))+\frac{\left(\ln \frac{T}{t_{m}}\right)^{\alpha-1}}{\Gamma(\alpha)} \sum_{i=1}^{m}\left[I_{i}\left(u\left(t_{i}\right)\right)\right.\right. \\
& \left.\left.+{ }_{H} J_{t_{i-1}}^{1} g\left(t_{i}, u\left(t_{i}\right), v\left(t_{i}\right)\right)+{ }_{H} J_{t_{i-1}}^{1} e\left(t_{i}, u\left(t_{i}\right), v\left(t_{i}\right)\right)\right]\right) .
\end{aligned}
$$


Thus, for $k=1,2, \ldots, m$, we have

$$
\begin{aligned}
u(t)= & { }_{H} J_{t_{k}}^{\alpha} g(t, u(t), v(t))+{ }_{H} J_{t_{k}}^{\alpha} e(t, u(t), v(t))+c_{1}\left(\ln \frac{t}{t_{k}}\right)^{\alpha-1} \\
& +\frac{1}{\Gamma(\alpha)} \sum_{i=1}^{k}\left[I_{i}\left(u\left(t_{i}\right)\right)+{ }_{H} J_{t_{i-1}}^{1} g\left(t_{i}, u\left(t_{i}\right), v\left(t_{i}\right)\right)\right. \\
& \left.+{ }_{H} J_{t_{i-1}}^{1} e\left(t_{i}, u\left(t_{i}\right), v\left(t_{i}\right)\right)\right]\left(\ln \frac{t}{t_{k}}\right)^{\alpha-1}, \quad t \in\left(t_{k}, t_{k+1}\right]
\end{aligned}
$$

Conversely, if $u(t)$ satisfies (2.2), it is easy to verify $u(t)$ satisfying (2.1). The proof is complete.

Similarly, we obtain the following lemma.

Lemma 2.6 Assume that the functions $f, h \in C[a, T]$ and $J_{k} \in C(\mathbb{R}, \mathbb{R})$. If $\rho \triangleq d \Gamma(\beta)-$ $\left(\ln \frac{T}{t_{m}}\right)^{\beta-1} \neq 0$, then, for given $u(t) \in \mathrm{PC}_{\alpha}[a, T]$, a function $v(t) \in \mathrm{PC}_{\beta}[a, T]$ is a solution of the impulsive Hadamard fractional differential equation

$$
\left\{\begin{array}{l}
\operatorname{RLH}_{t_{k}}^{\beta}\left[v(t)-{ }_{H} J_{t_{k}}^{\beta} f(t, u(t), v(t))\right]=h(t, u(t), v(t)), \quad 1<\beta<2, \\
\operatorname{RLH}_{t_{k}}^{\beta-1} v\left(t_{k}^{+}\right)-\mathrm{RLH}_{t_{k}}^{\beta-1} v\left(t_{k}^{-}\right)=J_{k}\left(v\left(t_{k}\right)\right), \quad k=1,2, \ldots, m, \\
d \cdot \mathrm{RLH}_{a} D_{a}^{\beta-1} v(a)=v(T)
\end{array}\right.
$$

if and only if $v(t) \in C[a, T] \cap \mathrm{PC}_{\beta}[a, T]$ is a solution of the integral equation

$$
\begin{aligned}
v(t)= & { }_{H} J_{t_{k}}^{\beta} h(t, u(t), v(t))+{ }_{H} J_{t_{k}}^{\beta} f(t, u(t), v(t))+d^{*}\left(\ln \frac{t}{t_{k}}\right)^{\beta-1} \\
& +\frac{\Lambda}{\Gamma(\beta)} \sum_{i=1}^{k}\left[J_{i}\left(v\left(t_{i}\right)\right)+{ }_{H} J_{t_{i-1}}^{1} h\left(t_{i}, u\left(t_{i}\right), v\left(t_{i}\right)\right)\right. \\
& \left.+{ }_{H} J_{t_{i-1}}^{1} f\left(t_{i}, u\left(t_{i}\right), v\left(t_{i}\right)\right)\right]\left(\ln \frac{t}{t_{k}}\right)^{\beta-1}, \quad t \in\left(t_{k}, t_{k+1}\right],
\end{aligned}
$$

where $k=0,1,2, \ldots, m, \Lambda=\left\{\begin{array}{l}0, t \in\left[a, t_{1}\right], \\ 1, t \in\left(t_{1}, T\right]\end{array}\right.$, and

$$
\begin{aligned}
d^{*}= & \frac{1}{\rho}\left({ }_{H} J_{t_{m}}^{\beta} h(T, u(T), v(T))+{ }_{H} J_{t_{m}}^{\beta} f(T, u(T), v(T))+\frac{\left(\ln \frac{T}{t_{m}}\right)^{\beta-1}}{\Gamma(\beta)} \sum_{i=1}^{m}\left[J_{i}\left(v\left(t_{i}\right)\right)\right.\right. \\
& \left.\left.+{ }_{H} J_{t_{i-1}}^{1} h\left(t_{i}, u\left(t_{i}\right), v\left(t_{i}\right)\right)+{ }_{H} J_{t_{i-1}}^{1} f\left(t_{i}, u\left(t_{i}\right), v\left(t_{i}\right)\right)\right]\right) .
\end{aligned}
$$

\section{Main results}

In this section, we shall employ Lemmas 2.3 and 2.4 to prove the existence of solutions to system (1.1). In the light of Lemmas 2.5 and 2.6, we define the operator $S: X=\mathrm{PC}_{\alpha} \times$ $\mathrm{PC}_{\beta} \rightarrow X$ by

$$
S(u, v)(t)=\left(S_{1}(u, v)(t), S_{2}(u, v)(t)\right)^{T}, \quad \forall(u, v) \in X, t \in[a, T],
$$


where

$$
\begin{aligned}
S_{1}(u, v)(t)= & { }_{H} J_{t_{k}}^{\alpha} g(t, u(t), v(t))+{ }_{H} J_{t_{k}}^{\alpha} e(t, u(t), v(t)) \\
& +c^{*}\left(\ln \frac{t}{t_{k}}\right)^{\alpha-1}+\frac{\Lambda}{\Gamma(\alpha)} \sum_{i=1}^{k}\left[I_{i}\left(u\left(t_{i}\right)\right)+{ }_{H} J_{t_{i-1}}^{1} g\left(t_{i}, u\left(t_{i}\right), v\left(t_{i}\right)\right)\right. \\
& \left.+{ }_{H} J_{t_{i-1}}^{1} e\left(t_{i}, u\left(t_{i}\right), v\left(t_{i}\right)\right)\right]\left(\ln \frac{t}{t_{k}}\right)^{\alpha-1}, \quad t \in\left(t_{k}, t_{k+1}\right], 0 \leq k \leq m,
\end{aligned}
$$

and

$$
\begin{aligned}
S_{2}(x, y)(t)= & { }_{H} J_{t_{k}}^{\beta} h(t, u(t), v(t))+{ }_{H} J_{t_{k}}^{\beta} f(t, u(t), v(t)) \\
& +d^{*}\left(\ln \frac{t}{t_{k}}\right)^{\beta-1}+\frac{\Lambda}{\Gamma(\beta)} \sum_{i=1}^{k}\left[J_{i}\left(v\left(t_{i}\right)\right)+{ }_{H} J_{t_{i-1}}^{1} h\left(t_{i}, u\left(t_{i}\right), v\left(t_{i}\right)\right)\right. \\
& \left.+{ }_{H} J_{t_{i-1}}^{1} f\left(t_{i}, u\left(t_{i}\right), v\left(t_{i}\right)\right)\right]\left(\ln \frac{t}{t_{k}}\right)^{\beta-1}, \quad t \in\left(t_{k}, t_{k+1}\right], 0 \leq k \leq m .
\end{aligned}
$$

Solving system (1.1) is equivalent to finding the fixed point of the operator $S$ defined by (3.1). Now we present and prove our main results.

Theorem 3.1 If the following conditions $\left(H_{1}\right)-\left(H_{3}\right)$ hold, then the Hadamard impulsive fractional differential coupled system (1.1) has a pair of unique solutions $\left(u^{*}(t), v^{*}(t)\right) \in$ $\mathrm{PC}_{\alpha} \times \mathrm{PC}_{\beta}$.

$\left(H_{1}\right)$ Let $e, f, g, h \in C[a, T], I_{k}, J_{k} \in C(\mathbb{R}, \mathbb{R}), k=1,2, \ldots, m$. For $u_{i}, v_{i} \in \mathbb{R}(i=1,2)$, there exist some positive constants $M_{i}, \bar{M}_{i}, N_{i}, \bar{N}_{i}(i=1,2), P_{k}$, and $Q_{k}(k=1,2, \ldots, m)$ such that

$$
\begin{aligned}
& \left|g\left(t, u_{1}, v_{1}\right)-e\left(t, u_{2}, v_{2}\right)\right| \leq M_{1}\left|u_{1}-u_{2}\right|+M_{2}\left|v_{1}-v_{2}\right|, \\
& \left|e\left(t, u_{1}, v_{1}\right)-g\left(t, u_{2}, v_{2}\right)\right| \leq N_{1}\left|u_{1}-u_{2}\right|+N_{2}\left|v_{1}-v_{2}\right|, \\
& \left|f\left(t, u_{1}, v_{1}\right)-f\left(t, u_{2}, v_{2}\right)\right| \leq \bar{M}_{1}\left|u_{1}-u_{2}\right|+\bar{M}_{2}\left|v_{1}-v_{2}\right|, \\
& \left|h\left(t, u_{1}, v_{1}\right)-h\left(t, u_{2}, v_{2}\right)\right| \leq \bar{N}_{1}\left|u_{1}-u_{2}\right|+\bar{N}_{2}\left|v_{1}-v_{2}\right|, \\
& \left|I_{k}\left(u_{1}\right)-I_{k}\left(v_{1}\right)\right| \leq P_{k}\left|u_{1}-v_{1}\right|, \\
& \left|J_{k}\left(u_{1}\right)-J_{k}\left(v_{1}\right)\right| \leq Q_{k}\left|u_{1}-v_{1}\right|, \quad k=1,2 \ldots, m .
\end{aligned}
$$

$\left(H_{2}\right) \delta \triangleq c \Gamma(\alpha)-\left(\ln \frac{T}{t_{m}}\right)^{\alpha-1}>0, \rho \triangleq d \Gamma(\beta)-\left(\ln \frac{T}{t_{m}}\right)^{\beta-1}>0$.

$\left(H_{3}\right)$

$$
\begin{aligned}
\kappa \triangleq & \frac{M_{1}+M_{2}+N_{1}+N_{2}}{\Gamma(\alpha)}\left(\ln \frac{T}{a}\right)^{\alpha} \\
& \times\left[1+\frac{1}{\delta}\left(\ln \frac{T}{a}\right)^{\alpha-1}+\frac{1}{\delta \Gamma(\alpha)}\left(\ln \frac{T}{a}\right)^{\alpha-1}+\frac{1}{\Gamma(\alpha)}\right] \\
& +\frac{\sum_{i=1}^{m} P_{i}}{\Gamma(\alpha)}\left(\ln \frac{T}{a}\right)^{\alpha-1}\left[1+\left(\ln \frac{T}{a}\right)^{\alpha-1}\right]<1,
\end{aligned}
$$




$$
\begin{aligned}
\varrho \triangleq & \frac{\bar{M}_{1}+\bar{M}_{2}+\bar{N}_{1}+\bar{N}_{2}}{\Gamma(\beta)}\left(\ln \frac{T}{a}\right)^{\beta} \\
& \times\left[1+\frac{1}{\rho}\left(\ln \frac{T}{a}\right)^{\beta-1}+\frac{1}{\rho \Gamma(\beta)}\left(\ln \frac{T}{a}\right)^{\beta-1}+\frac{1}{\Gamma(\beta)}\right] \\
& +\frac{\sum_{i=1}^{m} Q_{i}}{\Gamma(\beta)}\left(\ln \frac{T}{a}\right)^{\beta-1}\left[1+\left(\ln \frac{T}{a}\right)^{\beta-1}\right]<1 .
\end{aligned}
$$

Proof Now, we apply the Banach contraction principle to prove that $S: X \rightarrow X$ defined by (3.1) has a unique fixed point. We shall show that $S$ is a contraction. In fact, from (3.1) and conditions $\left(H_{1}\right)-\left(H_{2}\right)$, for $t \in J=[a, T],\left(u_{1}, v_{1}\right),\left(u_{2}, v_{2}\right) \in X$, we have

$$
\begin{aligned}
& \left|S_{1}\left(u_{1}, v_{1}\right)(t)-S_{1}\left(u_{2}, v_{2}\right)(t)\right| \\
& =\mid H J_{t_{k}}^{\alpha}\left[g\left(t, u_{1}(t), v_{1}(t)\right)-g\left(t, u_{2}(t), v_{2}(t)\right)\right]+{ }_{H} J_{t_{k}}^{\alpha}\left[e\left(t, u_{1}(t), v_{1}(t)\right)-e\left(t, u_{2}(t), v_{2}(t)\right)\right] \\
& +\frac{1}{\delta}\left[H J_{t_{m}}^{\alpha}\left[g\left(T, u_{1}(T), v_{1}(T)\right)-g\left(T, u_{2}(T), v_{2}(T)\right)\right]+{ }_{H} J_{t_{m}}^{\alpha}\left[e\left(T, u_{1}(T), v_{1}(T)\right)\right.\right. \\
& \left.-e\left(T, u_{2}(T), v_{2}(T)\right)\right]+\frac{\left(\ln \frac{T}{t_{m}}\right)^{\alpha-1}}{\Gamma(\alpha)} \sum_{i=1}^{m}\left(\left[I_{i}\left(u_{1}\left(t_{i}\right)\right)-I_{i}\left(u_{2}\left(t_{i}\right)\right)\right]\right. \\
& { }_{H} J_{t_{i-1}}^{1}\left[g\left(t_{i}, u_{1}\left(t_{i}\right), v_{1}\left(t_{i}\right)\right)-g\left(t_{i}, u_{2}\left(t_{i}\right), v_{2}\left(t_{i}\right)\right)\right] \\
& \left.\left.+{ }_{H} J_{t_{i-1}}^{1}\left[e\left(t_{i}, u_{1}\left(t_{i}\right), v_{1}\left(t_{i}\right)\right)-e\left(t_{i}, u_{2}\left(t_{i}\right), v_{2}\left(t_{i}\right)\right)\right]\right)\right]\left(\ln \frac{t}{t_{k}}\right)^{\alpha-1} \\
& +\frac{\Lambda}{\Gamma(\alpha)} \sum_{i=1}^{k}\left(\left[I_{i}\left(u_{1}\left(t_{i}\right)\right)-I_{i}\left(u_{2}\left(t_{i}\right)\right)\right]+{ }_{H} J_{t_{i-1}}^{1}\left[g\left(t_{i}, u_{1}\left(t_{i}\right), v_{2}\left(t_{i}\right)\right)-g\left(t_{i}, u_{1}\left(t_{i}\right), v_{2}\left(t_{i}\right)\right)\right]\right. \\
& \left.{ }_{H} J_{t_{i-1}}^{1}\left[e\left(t_{i}, u_{1}\left(t_{i}\right), v_{1}\left(t_{i}\right)\right)-e\left(t_{i}, u_{2}\left(t_{i}\right), v_{2}\left(t_{i}\right)\right)\right]\right)\left(\ln \frac{t}{t_{k}}\right)^{\alpha-1} \\
& \leq{ }_{H} J_{t_{k}}^{\alpha}\left|g\left(t, u_{1}(t), v_{1}(t)\right)-g\left(t, u_{2}(t), v_{2}(t)\right)\right|+{ }_{H} J_{t_{k}}^{\alpha}\left|e\left(t, u_{1}(t), v_{1}(t)\right)-e\left(t, u_{2}(t), v_{2}(t)\right)\right| \\
& +\frac{1}{\delta}\left[H J_{t_{m}}^{\alpha}\left|g\left(T, u_{1}(T), v_{1}(T)\right)-g\left(T, u_{2}(T), v_{2}(T)\right)\right|+{ }_{H} J_{t_{m}}^{\alpha} \mid e\left(T, u_{1}(T), v_{1}(T)\right)\right. \\
& -e\left(T, u_{2}(T), v_{2}(T)\right) \mid+\frac{\left(\ln \frac{T}{t_{m}}\right)^{\alpha-1}}{\Gamma(\alpha)} \sum_{i=1}^{m}\left(\mid I_{i}\left(u_{1}\left(t_{i}\right)\right)\right. \\
& -I_{i}\left(u_{2}\left(t_{i}\right)\right)\left|+{ }_{H} J_{t_{i-1}}^{1}\right| g\left(t_{i}, u_{1}\left(t_{i}\right), v_{1}\left(t_{i}\right)\right)-g\left(t_{i}, u_{2}\left(t_{i}\right), v_{2}\left(t_{i}\right)\right) \mid \\
& \left.\left.+{ }_{H} J_{t_{i-1}}^{1}\left|e\left(t_{i}, u_{1}\left(t_{i}\right), v_{1}\left(t_{i}\right)\right)-e\left(t_{i}, u_{2}\left(t_{i}\right), v_{2}\left(t_{i}\right)\right)\right|\right)\right]\left(\ln \frac{t}{t_{k}}\right)^{\alpha-1} \\
& +\frac{1}{\Gamma(\alpha)} \sum_{i=1}^{k}\left(\left|I_{i}\left(u_{1}\left(t_{i}\right)\right)-I_{i}\left(u_{2}\left(t_{i}\right)\right)\right|+{ }_{H} J_{t_{i-1}}^{1}\left|g\left(t_{i}, u_{1}\left(t_{i}\right), v_{2}\left(t_{i}\right)\right)-g\left(t_{i}, u_{1}\left(t_{i}\right), v_{2}\left(t_{i}\right)\right)\right|\right. \\
& \left.+{ }_{H} J_{t_{i-1}}^{1}\left|e\left(t_{i}, u_{1}\left(t_{i}\right), v_{1}\left(t_{i}\right)\right)-e\left(t_{i}, u_{2}\left(t_{i}\right), v_{2}\left(t_{i}\right)\right)\right|\right)\left(\ln \frac{t}{t_{k}}\right)^{\alpha-1} \mid \\
& \leq{ }_{H} J_{t_{k}}^{\alpha}\left[M_{1}\left|u_{1}(t)-u_{2}(t)\right|+M_{2}\left|v_{1}(t)-v_{2}(t)\right|\right]
\end{aligned}
$$




$$
\begin{aligned}
& +{ }_{H} J_{t_{k}}^{\alpha}\left[N_{1}\left|u_{1}(t)-u_{2}(t)\right|+N_{2}\left|v_{1}(t)-v_{2}(t)\right|\right] \\
& +\frac{1}{\delta}\left[{ }_{H} J_{t_{m}}^{\alpha}\left[M_{1}\left|u_{1}(T)-u_{2}(T)\right|+M_{2}\left|v_{1}(T)-v_{2}(T)\right|\right]+{ }_{H} J_{t_{m}}^{\alpha}\left[N_{1}\left|u_{1}(T)-u_{2}(T)\right|\right.\right. \\
& \left.+N_{2}\left|v_{1}(T)-v_{2}(T)\right|\right]+\frac{\left(\ln \frac{T}{t_{m}}\right)^{\alpha-1}}{\Gamma(\alpha)} \sum_{i=1}^{m}\left(P_{i}\left|u_{1}\left(t_{i}\right)-u_{2}\left(t_{i}\right)\right|\right. \\
& +{ }_{H} J_{t_{i-1}}^{1}\left[M_{1}\left|u_{1}\left(t_{i}\right)-u_{2}\left(t_{i}\right)\right|+M_{2}\left|v_{1}\left(t_{i}\right)-v_{2}\left(t_{i}\right)\right|\right] \\
& \left.\left.+{ }_{H} J_{t_{i-1}}^{1}\left[N_{1}\left|u_{1}\left(t_{i}\right)-u_{2}\left(t_{i}\right)\right|+N_{2}\left|v_{1}\left(t_{i}\right)-v_{2}\left(t_{i}\right)\right|\right]\right)\right]\left(\ln \frac{t}{t_{k}}\right)^{\alpha-1} \\
& +\frac{1}{\Gamma(\alpha)} \sum_{i=1}^{k}\left(P_{i}\left|u_{1}\left(t_{i}\right)-u_{2}\left(t_{i}\right)\right|+{ }_{H} J_{t_{i-1}}^{1}\left[M_{1}\left|u_{1}\left(t_{i}\right)-u_{2}\left(t_{i}\right)\right|+M_{2}\left|v_{1}\left(t_{i}\right)-v_{2}\left(t_{i}\right)\right|\right]\right. \\
& +{ }_{H} J_{t_{i-1}}^{1}\left[N_{1}\left|u_{1}\left(t_{i}\right)-u_{2}\left(t_{i}\right)\right|+N_{2}\left[\left|v_{1}\left(t_{i}\right)-v_{2}\left(t_{i}\right)\right|\right]\right)\left(\ln \frac{t}{t_{k}}\right)^{\alpha-1} \\
& \leq{ }_{H} J_{t_{k}}^{\alpha}\left[M_{1}\left\|u_{1}-u_{2}\right\|_{\mathrm{PC}_{\alpha}}+M_{2}\left\|v_{1}-v_{2}\right\|_{\mathrm{PC}_{\beta}}\right] \\
& +{ }_{H} J_{t_{k}}^{\alpha}\left[N_{1}\left\|u_{1}-u_{2}\right\|_{\mathrm{PC}_{\alpha}}+N_{2}\left\|v_{1}-v_{2}\right\|_{\mathrm{PC}_{\beta}}\right] \\
& +\frac{1}{\delta}\left[{ }_{H} J_{t_{m}}^{\alpha}\left[M_{1}\left\|u_{1}-u_{2}\right\|_{\mathrm{PC}_{\alpha}}+M_{2}\left\|v_{1}-v_{2}\right\|_{\mathrm{PC}_{\beta}}\right]+{ }_{H} J_{t_{m}}^{\alpha}\left[N_{1}\left\|u_{1}-u_{2}\right\|_{\mathrm{PC}_{\alpha}}\right.\right. \\
& \left.+N_{2}\left\|v_{1}-v_{2}\right\|_{\mathrm{PC}_{\beta}}\right]+\frac{\left(\ln \frac{T}{t_{m}}\right)^{\alpha-1}}{\Gamma(\alpha)} \sum_{i=1}^{m}\left(P_{i}\left\|u_{1}-u_{2}\right\|_{\mathrm{PC}_{\alpha}}+{ }_{H} J_{t_{i-1}}^{1}\left[M_{1}\left\|u_{1}-u_{2}\right\|_{\mathrm{PC}_{\alpha}}\right.\right. \\
& \left.\left.\left.+M_{2}\left\|v_{1}-v_{2}\right\|_{\mathrm{PC}_{\beta}}\right]+{ }_{H} J_{t_{i-1}}^{1}\left[N_{1}\left\|u_{1}-u_{2}\right\|_{\mathrm{PC}_{\alpha}}+N_{2}\left\|v_{1}-v_{2}\right\|_{\mathrm{PC}_{\beta}}\right]\right)\right]\left(\ln \frac{t}{t_{k}}\right)^{\alpha-1} \\
& +\frac{1}{\Gamma(\alpha)} \sum_{i=1}^{k}\left(P_{i}\left\|u_{1}-u_{2}\right\|_{\mathrm{PC}_{\alpha}}+{ }_{H} J_{t_{i-1}}^{1}\left[M_{1}\left\|u_{1}-u_{2}\right\|_{\mathrm{PC}_{\alpha}}+M_{2}\left\|v_{1}-v_{2}\right\|_{\mathrm{PC}_{\beta}}\right]\right. \\
& +{ }_{H} J_{t_{i-1}}^{1}\left[N_{1}\left\|u_{1}-u_{2}\right\|_{\mathrm{PC}_{\alpha}}+N_{2}\left[\left\|v_{1}-v_{2}\right\|_{\mathrm{PC}_{\beta}}\right]\right)\left(\ln \frac{t}{t_{k}}\right)^{\alpha-1} \\
& \leq\left(M_{1}+M_{2}+N_{1}+N_{2}\right)\left\|\left(u_{1}-u_{2}, v_{1}-v_{2}\right)\right\| \frac{1}{\Gamma(\alpha)} \int_{t_{k}}^{t}\left(\ln \frac{t}{s}\right)^{\alpha-1} \frac{d s}{s} \\
& +\frac{1}{\delta}\left[\left(M_{1}+M_{2}+N_{1}+N_{2}\right)\left\|\left(u_{1}-u_{2}, v_{1}-v_{2}\right)\right\| \frac{1}{\Gamma(\alpha)} \int_{t_{m}}^{T}\left(\ln \frac{T}{s}\right)^{\alpha-1} \frac{d s}{s}\right. \\
& +\frac{\left(\ln \frac{T}{t_{m}}\right)^{\alpha-1}}{\Gamma(\alpha)} \sum_{i=1}^{m}\left(P_{i}\left\|\left(u_{1}-u_{2}, v_{1}-v_{2}\right)\right\|\right. \\
& +\left(M_{1}+M_{2}+N_{1}+N_{2}\right)\left\|\left(u_{1}-u_{2}, v_{1}-v_{2}\right)\right\| \\
& \left.\left.\times \frac{1}{\Gamma(\alpha)} \int_{t_{i-1}}^{t_{i}} \frac{d s}{s}\right)\right]\left(\ln \frac{t}{t_{k}}\right)^{\alpha-1}+\frac{1}{\Gamma(\alpha)} \sum_{i=1}^{k}\left(P_{i}\left\|\left(u_{1}-u_{2}, v_{1}-v_{2}\right)\right\|\right. \\
& \left.+\left(M_{1}+M_{2}+N_{1}+N_{2}\right)\left\|\left(u_{1}-u_{2}, v_{1}-v_{2}\right)\right\| \frac{1}{\Gamma(\alpha)} \int_{t_{i-1}}^{t_{i}} \frac{d s}{s}\right)\left(\ln \frac{t}{t_{k}}\right)^{\alpha-1}
\end{aligned}
$$




$$
\begin{aligned}
\leq & \frac{M_{1}+M_{2}+N_{1}+N_{2}}{\Gamma(\alpha)}\left(\ln \frac{T}{a}\right)^{\alpha}\left\|\left(u_{1}-u_{2}, v_{1}-v_{2}\right)\right\| \\
& +\frac{1}{\delta}\left[\frac{M_{1}+M_{2}+N_{1}+N_{2}}{\Gamma(\alpha)}\left(\ln \frac{T}{a}\right)^{\alpha}\left\|\left(u_{1}-u_{2}, v_{1}-v_{2}\right)\right\|\right. \\
& \left.+\frac{\left(\ln \frac{T}{a}\right)^{\alpha-1}}{\Gamma(\alpha)}\left(\sum_{i=1}^{m} P_{i}+\frac{M_{1}+M_{2}+N_{1}+N_{2}}{\Gamma(\alpha)} \ln \frac{T}{a}\right)\left\|\left(u_{1}-u_{2}, v_{1}-v_{2}\right)\right\|\right]\left(\ln \frac{T}{a}\right)^{\alpha-1} \\
& +\frac{1}{\Gamma(\alpha)}\left(\sum_{i=1}^{k} P_{i}+\frac{M_{1}+M_{2}+N_{1}+N_{2}}{\Gamma(\alpha)} \ln \frac{T}{a}\right)\left(\ln \frac{T}{a}\right)^{\alpha-1}\left\|\left(u_{1}-u_{2}, v_{1}-v_{2}\right)\right\| \\
\leq & \left\{\frac{M_{1}+M_{2}+N_{1}+N_{2}}{\Gamma(\alpha)}\left(\ln \frac{T}{a}\right)^{\alpha}\left[1+\frac{1}{\delta}\left(\ln \frac{T}{a}\right)^{\alpha-1}+\frac{1}{\delta \Gamma(\alpha)}\left(\ln \frac{T}{a}\right)^{\alpha-1}+\frac{1}{\Gamma(\alpha)}\right]\right. \\
& \left.+\frac{\sum_{i=1}^{m} P_{i}}{\Gamma(\alpha)}\left(\ln \frac{T}{a}\right)^{\alpha-1}\left[1+\left(\ln \frac{T}{a}\right)^{\alpha-1}\right]\right\}\left\|\left(u_{1}-u_{2}, v_{1}-v_{2}\right)\right\| \\
= & \kappa\left\|\left(u_{1}-u_{2}, v_{1}-v_{2}\right)\right\| .
\end{aligned}
$$

Similarly, we derive

$$
\begin{aligned}
& \left|S_{2}\left(u_{1}, v_{1}\right)(t)-S_{2}\left(u_{2}, v_{2}\right)(t)\right| \\
& \leq\left\{\frac{\bar{M}_{1}+\bar{M}_{2}+\bar{N}_{1}+\bar{N}_{2}}{\Gamma(\beta)}\left(\ln \frac{T}{a}\right)^{\beta}\left[1+\frac{1}{\rho}\left(\ln \frac{T}{a}\right)^{\beta-1}+\frac{1}{\rho \Gamma(\beta)}\left(\ln \frac{T}{a}\right)^{\beta-1}+\frac{1}{\Gamma(\beta)}\right]\right. \\
& \left.\quad+\frac{\sum_{i=1}^{m} Q_{i}}{\Gamma(\beta)}\left(\ln \frac{T}{a}\right)^{\beta-1}\left[1+\left(\ln \frac{T}{a}\right)^{\beta-1}\right]\right\}\left\|\left(u_{1}-u_{2}, v_{1}-v_{2}\right)\right\| \\
& =\varrho\left\|\left(u_{1}-u_{2}, v_{1}-v_{2}\right)\right\| .
\end{aligned}
$$

According to $\left(H_{3}\right),(3.2)$, and (3.3), we get

$$
\begin{aligned}
\left\|S\left(u_{1}, v_{1}\right)-S\left(u_{2}, v_{2}\right)\right\| & =\max \left\{\left\|S_{1}\left(u_{1}, v_{1}\right)-S_{1}\left(u_{2}, v_{2}\right)\right\|_{\mathrm{PC}_{\alpha}},\left\|S_{2}\left(u_{1}, v_{1}\right)-S_{2}\left(u_{2}, v_{2}\right)\right\|_{\mathrm{PC}_{\beta}}\right\} \\
& \leq \max \{\kappa, \varrho\}\left\|\left(u_{1}, v_{1}\right)\right\|<\left\|\left(u_{1}, v_{1}\right)\right\| .
\end{aligned}
$$

Therefore, (3.4) means that $S: X \rightarrow X$ defined by (3.1) is a contraction. According to Lemma 2.3, $S$ has a unique fixed point $\left(u^{*}(t), v^{*}(t)\right) \in X$, which is a pair of unique solutions of system (1.1). The proof of Theorem 3.1 is completed.

Theorem 3.2 Let e,f, $g, h \in C[a, T]), I_{k}, J_{k} \in C(\mathbb{R}, \mathbb{R}), k=1,2, \ldots, m$. Assume that there exist some positive constants $L_{1}, L_{2}, \bar{L}_{1}, \bar{L}_{2}, O_{k}$, and $\bar{O}_{k}(k=1,2, \ldots, m)$ such that

$\left(H_{4}\right)|e(t, u, v)| \leq L_{1},|g(t, u, v)| \leq L_{2},|f(t, u, v)| \leq \bar{L}_{1},|h(t, u, v)| \leq \bar{L}_{2},\left|I_{k}(u)\right| \leq O_{k}$ and $\left|J_{k}(v)\right| \leq \bar{O}_{k}(k=1,2, \ldots, m)$ for all $t \in(a, T], u, v \in \mathbb{R}$.

If $\left(H_{2}\right)$ and $\left(H_{4}\right)$ hold, then the Hadamard impulsive fractional differential coupled system (1.1) has at least a pair of solutions $\left(u^{*}(t), v^{*}(t)\right)$.

Proof Define the operator $S: X \rightarrow X$ as (3.1). In order to apply the Leray-Schauder alternative theorem, we need first to prove that $S$ is completely continuous. Indeed, in view of the continuities of $e, g, f, h, I_{k}$, and $J_{k}$, it is easy to know that $T$ is continuous. 
Now we show that the operator $S$ is uniformly bounded. Let $r>0, B_{r}=\{(u, v) \in$ $X,\|(u, v)\| \leq r\}$ be any bounded subset of $X$. For all $(u, v) \in B_{r}, t \in[a, T]$, it follows from $\left(H_{4}\right)$ that

$$
\begin{aligned}
& \left|S_{1}(u, v)(t)\right| \\
& =\mid H J_{t_{k}}^{\alpha} g(t, u(t), v(t))+{ }_{H} J_{t_{k}}^{\alpha} e(t, u(t), v(t))+\frac{1}{\delta}\left({ }_{H} J_{t_{m}}^{\alpha} g(T, u(T), v(T))\right. \\
& +{ }_{H} J_{t_{m}}^{\alpha} e(T, u(T), v(T))+\frac{\left(\ln \frac{T}{t_{m}}\right)^{\alpha-1}}{\Gamma(\alpha)} \sum_{i=1}^{m}\left[I_{i}\left(u\left(t_{i}\right)\right)+{ }_{H} J_{t_{i-1}}^{1} g\left(t_{i}, u\left(t_{i}\right), v\left(t_{i}\right)\right)\right. \\
& \left.\left.{ }_{H} J_{t_{i-1}}^{1} e\left(t_{i}, u\left(t_{i}\right), v\left(t_{i}\right)\right)\right]\right)\left(\ln \frac{t}{t_{k}}\right)^{\alpha-1}+\frac{\Lambda}{\Gamma(\alpha)} \sum_{i=1}^{k}\left[I_{i}\left(u\left(t_{i}\right)\right)\right. \\
& \left.+{ }_{H} J_{t_{i-1}}^{1} g\left(t_{i}, u\left(t_{i}\right), v\left(t_{i}\right)\right)+{ }_{H} J_{t_{i-1}}^{1} e\left(t_{i}, u\left(t_{i}\right), v\left(t_{i}\right)\right)\right]\left(\ln \frac{t}{t_{k}}\right)^{\alpha-1} \\
& \leq{ }_{H} J_{t_{k}}^{\alpha}|g(t, u(t), v(t))|+{ }_{H} J_{t_{k}}^{\alpha}|e(t, u(t), v(t))|+\frac{1}{\delta}\left({ }_{H} J_{t_{m}}^{\alpha}|g(T, u(T), v(T))|\right. \\
& +{ }_{H} J_{t_{m}}^{\alpha}|e(T, u(T), v(T))|+\frac{\left(\ln \frac{T}{t_{m}}\right)^{\alpha-1}}{\Gamma(\alpha)} \sum_{i=1}^{m}\left[\left|I_{i}\left(u\left(t_{i}\right)\right)\right|+{ }_{H} J_{t_{i-1}}^{1}\left|g\left(t_{i}, u\left(t_{i}\right), v\left(t_{i}\right)\right)\right|\right. \\
& \left.\left.+{ }_{H} J_{t_{i-1}}^{1}\left|e\left(t_{i}, u\left(t_{i}\right), v\left(t_{i}\right)\right)\right|\right]\right)\left(\ln \frac{t}{t_{k}}\right)^{\alpha-1}+\frac{1}{\Gamma(\alpha)} \sum_{i=1}^{k}\left[\left|I_{i}\left(u\left(t_{i}\right)\right)\right|\right. \\
& \left.+{ }_{H} J_{t_{i-1}}^{1}\left|g\left(t_{i}, u\left(t_{i}\right), v\left(t_{i}\right)\right)\right|+{ }_{H} J_{t_{i-1}}^{1}\left|e\left(t_{i}, u\left(t_{i}\right), v\left(t_{i}\right)\right)\right|\right]\left(\ln \frac{t}{t_{k}}\right)^{\alpha-1} \\
& \leq \frac{L_{1}+L_{2}}{\Gamma(\alpha)} \int_{t_{k}}^{t}\left(\ln \frac{t}{s}\right)^{\alpha-1} \frac{d s}{s}+\frac{1}{\delta}\left(\frac{L_{1}+L_{2}}{\Gamma(\alpha)} \int_{t_{m}}^{T}\left(\ln \frac{T}{s}\right)^{\alpha-1} \frac{d s}{s}+\frac{\left(\ln \frac{T}{t_{m}}\right)^{\alpha-1}}{\Gamma(\alpha)} \sum_{i=1}^{m}\left[O_{i}\right.\right. \\
& \left.\left.+\frac{L_{1}+L_{2}}{\Gamma(\alpha)} \int_{t_{i-1}}^{t_{i}} \frac{d s}{s}\right]\right)\left(\ln \frac{t}{t_{k}}\right)^{\alpha-1}+\frac{1}{\Gamma(\alpha)} \sum_{i=1}^{k}\left[O_{i}+\frac{L_{1}+L_{2}}{\Gamma(\alpha)} \int_{t_{i-1}}^{t_{i}} \frac{d s}{s}\right]\left(\ln \frac{t}{t_{k}}\right)^{\alpha-1} \\
& \leq \frac{L_{1}+L_{2}}{\Gamma(\alpha)} \int_{a}^{T}\left(\ln \frac{T}{a}\right)^{\alpha-1} \frac{d s}{s}+\frac{1}{\delta}\left(\frac{L_{1}+L_{2}}{\Gamma(\alpha)} \int_{a}^{T}\left(\ln \frac{T}{a}\right)^{\alpha-1} \frac{d s}{s}\right. \\
& \left.+\frac{\left(\ln \frac{T}{a}\right)^{\alpha-1}}{\Gamma(\alpha)}\left[\sum_{i=1}^{m} O_{i}+\frac{L_{1}+L_{2}}{\Gamma(\alpha)} \int_{a}^{T} \frac{d s}{s}\right]\right)\left(\ln \frac{T}{a}\right)^{\alpha-1} \\
& +\frac{1}{\Gamma(\alpha)}\left[\sum_{i=1}^{m} O_{i}+\frac{L_{1}+L_{2}}{\Gamma(\alpha)} \int_{a}^{T} \frac{d s}{s}\right]\left(\ln \frac{T}{a}\right)^{\alpha-1} \\
& =\frac{L_{1}+L_{2}}{\Gamma(\alpha)}\left(\ln \frac{T}{a}\right)^{\alpha}\left[1+\frac{1}{\delta}\left(\ln \frac{T}{a}\right)^{\alpha-1}+\frac{1}{\delta \Gamma(\alpha)}\left(\ln \frac{T}{a}\right)^{\alpha-1}+\frac{1}{\Gamma(\alpha)}\right] \\
& +\frac{\sum_{i=1}^{m} P_{i}}{\Gamma(\alpha)}\left(\ln \frac{T}{a}\right)^{\alpha-1}\left[1+\left(\ln \frac{T}{a}\right)^{\alpha-1}\right] \triangleq A .
\end{aligned}
$$


Similarly, we have

$$
\begin{aligned}
\left|S_{2}(u, v)(t)\right| \leq & \frac{\bar{L}_{1}+\bar{L}_{2}}{\Gamma(\beta)}\left(\ln \frac{T}{a}\right)^{\beta}\left[1+\frac{1}{\rho}\left(\ln \frac{T}{a}\right)^{\beta-1}+\frac{1}{\rho \Gamma(\beta)}\left(\ln \frac{T}{a}\right)^{\beta-1}+\frac{1}{\Gamma(\beta)}\right] \\
& +\frac{\sum_{i=1}^{m} \bar{O}_{i}}{\Gamma(\beta)}\left(\ln \frac{T}{a}\right)^{\beta-1}\left[1+\left(\ln \frac{T}{a}\right)^{\beta-1}\right] \triangleq B .
\end{aligned}
$$

From (3.5) and (3.6), we know that $S$ is uniformly bounded.

Next, we show that the operator $S$ is equicontinuous. In fact, for any $\tau_{1}, \tau_{2} \in[a, T], \tau_{1}<$ $\tau_{2}, \tau_{2}-\tau_{1}$ is small enough such that $\tau_{1}, \tau_{2} \in\left[t_{k}, t_{k+1}\right], k=0,1,2, \ldots, m$, we have

$$
\begin{aligned}
& \left|S_{1}(u, v)\left(\tau_{2}\right)-S_{1}(u, v)\left(\tau_{1}\right)\right| \\
& =\mid H J_{t_{k}}^{\alpha} g\left(\tau_{2}, u\left(\tau_{2}\right), v\left(\tau_{2}\right)\right)-{ }_{H} J_{t_{k}}^{\alpha} g\left(\tau_{1}, u\left(\tau_{1}\right), v\left(\tau_{1}\right)\right) \\
& { }_{{ }_{H}} J_{t_{k}}^{\alpha} e\left(\tau_{2}, u\left(\tau_{2}\right), v\left(\tau_{2}\right)\right)-{ }_{H} J_{t_{k}}^{\alpha} e\left(\tau_{1}, u\left(\tau_{1}\right), v\left(\tau_{1}\right)\right) \\
& +c^{*}\left[\left(\ln \frac{\tau_{2}}{t_{k}}\right)^{\alpha-1}-\left(\ln \frac{\tau_{1}}{t_{k}}\right)^{\alpha-1}\right]+\frac{\Lambda}{\Gamma(\alpha)} \sum_{i=1}^{k}\left[I_{i}\left(u\left(t_{i}\right)\right)+{ }_{H} J_{t_{i-1}}^{1} g\left(t_{i}, u\left(t_{i}\right), v\left(t_{i}\right)\right)\right. \\
& \left.+{ }_{H} J_{t_{i-1}}^{1} e\left(t_{i}, u\left(t_{i}\right), v\left(t_{i}\right)\right)\right]\left[\left(\ln \frac{\tau_{2}}{t_{k}}\right)^{\alpha-1}-\left(\ln \frac{\tau_{1}}{t_{k}}\right)^{\alpha-1}\right] \\
& =\mid \frac{1}{\Gamma(\alpha)} \int_{t_{k}}^{\tau_{2}}\left(\ln \frac{\tau_{2}}{s}\right)^{\alpha-1} g(s, u(s), v(s)) \frac{d s}{s}-\frac{1}{\Gamma(\alpha)} \int_{t_{k}}^{\tau_{1}}\left(\ln \frac{\tau_{1}}{s}\right)^{\alpha-1} g(s, u(s), v(s)) \frac{d s}{s} \\
& +\frac{1}{\Gamma(\alpha)} \int_{t_{k}}^{\tau_{2}}\left(\ln \frac{\tau_{2}}{s}\right)^{\alpha-1} e(s, u(s), v(s)) \frac{d s}{s}-\frac{1}{\Gamma(\alpha)} \int_{t_{k}}^{\tau_{1}}\left(\ln \frac{\tau_{1}}{s}\right)^{\alpha-1} e(s, u(s), v(s)) \frac{d s}{s} \\
& +c^{*}\left[\left(\ln \frac{\tau_{2}}{t_{k}}\right)^{\alpha-1}-\left(\ln \frac{\tau_{1}}{t_{k}}\right)^{\alpha-1}\right]+\frac{\Lambda}{\Gamma(\alpha)} \sum_{i=1}^{k}\left[I_{i}\left(u\left(t_{i}\right)\right)+{ }_{H} J_{t_{i-1}}^{1} g\left(t_{i}, u\left(t_{i}\right), v\left(t_{i}\right)\right)\right. \\
& \left.+{ }_{H} J_{t_{i-1}}^{1} e\left(t_{i}, u\left(t_{i}\right), v\left(t_{i}\right)\right)\right]\left[\left(\ln \frac{\tau_{2}}{t_{k}}\right)^{\alpha-1}-\left(\ln \frac{\tau_{1}}{t_{k}}\right)^{\alpha-1}\right] \mid \\
& \leq \frac{1}{\Gamma(\alpha)}\left\{\int_{t_{k}}^{\tau_{1}}\left[\left(\ln \frac{\tau_{2}}{s}\right)^{\alpha-1}-\left(\ln \frac{\tau_{1}}{s}\right)^{\alpha-1}\right]|g(s, u(s), v(s))| \frac{d s}{s}+\int_{\tau_{1}}^{\tau_{2}}\left(\ln \frac{\tau_{2}}{s}\right)^{\alpha-1}\right. \\
& \times|g(s, u(s), v(s))| \frac{d s}{s}+\int_{t_{k}}^{\tau_{1}}\left[\left(\ln \frac{\tau_{2}}{s}\right)^{\alpha-1}-\left(\ln \frac{\tau_{1}}{s}\right)^{\alpha-1}\right]|e(s, u(s), v(s))| \frac{d s}{s} \\
& \left.+\int_{\tau_{1}}^{\tau_{2}}\left(\ln \frac{\tau_{2}}{s}\right)^{\alpha-1}|e(s, u(s), v(s))| \frac{d s}{s}\right\}+\left|c^{*}\right|\left[\left(\ln \frac{\tau_{2}}{t_{k}}\right)^{\alpha-1}-\left(\ln \frac{\tau_{1}}{t_{k}}\right)^{\alpha-1}\right] \\
& +\frac{1}{\Gamma(\alpha)} \sum_{i=1}^{m}\left[\left|I_{i}\left(u\left(t_{i}\right)\right)\right|+{ }_{H} J_{t_{i-1}}^{1}\left|g\left(t_{i}, u\left(t_{i}\right), v\left(t_{i}\right)\right)\right|+{ }_{H} J_{t_{i-1}}^{1}\left|e\left(t_{i}, u\left(t_{i}\right), v\left(t_{i}\right)\right)\right|\right] \\
& \times\left[\left(\ln \frac{\tau_{2}}{t_{k}}\right)^{\alpha-1}-\left(\ln \frac{\tau_{1}}{t_{k}}\right)^{\alpha-1}\right] \\
& \leq \frac{1}{\Gamma(\alpha)}\left\{L_{1} \int_{t_{k}}^{\tau_{1}}\left[\left(\ln \frac{\tau_{2}}{s}\right)^{\alpha-1}-\left(\ln \frac{\tau_{1}}{s}\right)^{\alpha-1}\right] \frac{d s}{s}+L_{1} \int_{\tau_{1}}^{\tau_{2}}\left(\ln \frac{\tau_{2}}{s}\right)^{\alpha-1} \frac{d s}{s}\right.
\end{aligned}
$$




$$
\begin{aligned}
& \left.+L_{2} \int_{t_{k}}^{\tau_{1}}\left[\left(\ln \frac{\tau_{2}}{s}\right)^{\alpha-1}-\left(\ln \frac{\tau_{1}}{s}\right)^{\alpha-1}\right] \frac{d s}{s}+L_{2} \int_{\tau_{1}}^{\tau_{2}}\left(\ln \frac{\tau_{2}}{s}\right)^{\alpha-1} \frac{d s}{s}\right\} \\
& +\left|c^{*}\right|\left[\left(\ln \frac{\tau_{2}}{t_{k}}\right)^{\alpha-1}-\left(\ln \frac{\tau_{1}}{t_{k}}\right)^{\alpha-1}\right]+\frac{1}{\Gamma(\alpha)}\left[\sum_{i=1}^{m} O_{i}+\frac{L_{1}}{\Gamma(\alpha)} \int_{a}^{t_{m}} \frac{d s}{s}\right. \\
& \left.+\frac{L_{2}}{\Gamma(\alpha)} \int_{a}^{t_{m}} \frac{d s}{s}\right]\left[\left(\ln \frac{\tau_{2}}{t_{k}}\right)^{\alpha-1}-\left(\ln \frac{\tau_{1}}{t_{k}}\right)^{\alpha-1}\right] \rightarrow 0, \quad \text { as } \tau_{1} \rightarrow \tau_{2} .
\end{aligned}
$$

We similarly get

$$
\left|S_{2}(u, v)\left(\tau_{2}\right)-S_{2}(u, v)\left(\tau_{1}\right)\right| \rightarrow 0, \quad \text { as } \tau_{1} \rightarrow \tau_{2}
$$

(3.7) and (3.8) mean that $S$ is equicontinuous. By the Ascoli-Arzelá theorem, we know that $S$ is completely continuous.

Finally, we prove that the set $\varepsilon(S)=\{(u, v) \in X \mid(u, v)=\lambda S(u, v), 0<\lambda<1\}$ is bounded. Let $(u, v) \in \varepsilon(S)$, then $(u, v)=\lambda S(u, v)$, for any $t \in[a, T]$, we have

$$
u(t)=\lambda S_{1}(u, v)(t), \quad v(t)=\lambda S_{2}(u, v)(t)
$$

For $t \in\left(t_{k}, t_{k+1}\right], k=0,1,2, \ldots, m$, it follows from (3.5) and (3.6) that

$$
|u(t)|=\left|\lambda S_{1}(u, v)(t)\right|=\lambda\left|S_{1}(u, v)(t)\right| \leq \lambda A
$$

and

$$
|v(t)|=\left|\lambda S_{2}(u, v)(t)\right|=\lambda\left|S_{2}(u, v)(t)\right| \leq \lambda B
$$

(3.9) and (3.10) implicate that $\varepsilon(S)$ is bounded for any $t \in[a, T]$. In view of Lemma 2.4, the operator $S$ defined by (3.1) has at least one fixed point. Hence, the Hadamard impulsive fractional differential coupled system (1.1) has at least a pair of solutions $\left(u^{*}(t), v^{*}(t)\right)$. The proof is completed.

\section{Illustrative examples}

Consider the nonlinear Hadamard fractional integro-differential coupled system with impulses as follows:

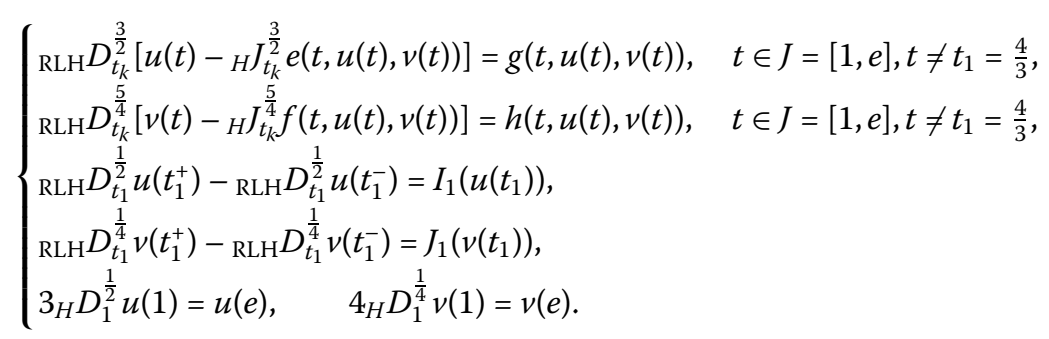

Case 1 Take $g(t, u, v)=\frac{\left(u^{2}+v^{3}\right) \cos 2 t}{180}, e(t, u, v)=\frac{(u+v) \sin t}{50}, f(t, u, v)=\frac{e^{-t}(\sqrt[3]{u}+\sqrt[5]{v})}{150}, h(t, u, v)=$ $\frac{\sin u \cos v \arcsin t}{20 \pi}, I_{1}(u)=\frac{u^{2}}{10}, J_{1}(v)=\frac{u^{4}}{20}$. Obviously, $e, g, f, h \in C[1, e], I_{1}, J_{1} \in C(\mathbb{R}, \mathbb{R})$. By the simple calculation, we have

$$
\left|g\left(t, u_{1}, v_{1}\right)-g\left(t, u_{2}, v_{2}\right)\right| \leq \frac{1}{90}\left|u_{1}-u_{2}\right|+\frac{1}{60}\left|v_{1}-v_{2}\right|,
$$




$$
\begin{aligned}
& \left|e\left(t, u_{1}, v_{1}\right)-e\left(t, u_{2}, v_{2}\right)\right| \leq \frac{1}{50}\left|u_{1}-u_{2}\right|+\frac{1}{50}\left|v_{1}-v_{2}\right|, \\
& \left|f\left(t, u_{1}, v_{1}\right)-f\left(t, u_{2}, v_{2}\right)\right| \leq \frac{1}{50}\left|u_{1}-u_{2}\right|+\frac{1}{30}\left|v_{1}-v_{2}\right|, \\
& \left|h\left(t, u_{1}, v_{1}\right)-h\left(t, u_{2}, v_{2}\right)\right| \leq \frac{1}{10}\left|u_{1}-u_{2}\right|+\frac{1}{10}\left|v_{1}-v_{2}\right|, \\
& \left|I_{1}\left(u_{1}\right)-e\left(u_{2}\right)\right| \leq \frac{1}{5}\left|u_{1}-u_{2}\right|, \quad\left|J_{1}\left(v_{1}\right)-J_{1}\left(v_{2}\right)\right| \leq \frac{1}{5}\left|v_{1}-v_{2}\right|,
\end{aligned}
$$

that is, $M_{1}=\frac{1}{90}, M_{2}=\frac{1}{60}, N_{1}=N_{2}=\frac{1}{50}, \bar{M}_{1}=\frac{1}{50}, \bar{M}_{2}=\frac{1}{30}, \bar{N}_{1}=\bar{N}_{1}=\frac{1}{10}, P_{1}=Q_{1}=\frac{1}{5}$. Thus, we obtain

$$
\begin{aligned}
\delta= & c \Gamma(\alpha)-\left(\ln \frac{T}{t_{1}}\right)^{\alpha-1} \approx 1.8147>0, \quad \rho=d \Gamma(\beta)-\left(\ln \frac{T}{t_{1}}\right)^{\beta-1} \approx 2.7069>0, \\
\kappa= & \frac{M_{1}+M_{2}+N_{1}+N_{2}}{\Gamma(\alpha)}\left(\ln \frac{T}{a}\right)^{\alpha}\left[1+\frac{1}{\delta}\left(\ln \frac{T}{a}\right)^{\alpha-1}+\frac{1}{\delta \Gamma(\alpha)}\left(\ln \frac{T}{a}\right)^{\alpha-1}+\frac{1}{\Gamma(\alpha)}\right] \\
& +\frac{\sum_{i=1}^{m} P_{i}}{\Gamma(\alpha)}\left(\ln \frac{T}{a}\right)^{\alpha-1}\left[1+\left(\ln \frac{T}{a}\right)^{\alpha-1}\right] \approx 0.7038<1, \\
\varrho= & \frac{\bar{M}_{1}+\bar{M}_{2}+\bar{N}_{1}+\bar{N}_{2}}{\Gamma(\beta)}\left(\ln \frac{T}{a}\right)^{\beta}\left[1+\frac{1}{\rho}\left(\ln \frac{T}{a}\right)^{\beta-1}+\frac{1}{\rho \Gamma(\beta)}\left(\ln \frac{T}{a}\right)^{\beta-1}+\frac{1}{\Gamma(\beta)}\right] \\
& +\frac{\sum_{i=1}^{m} Q_{i}}{\Gamma(\beta)}\left(\ln \frac{T}{a}\right)^{\beta-1}\left[1+\left(\ln \frac{T}{a}\right)^{\beta-1}\right] \approx 0.7379<1 .
\end{aligned}
$$

Therefore, conditions $\left(H_{1}\right)-\left(H_{3}\right)$ of Theorem 3.1 hold. Then (4.1) has a pair of unique solutions $\left(u^{*}(t), v^{*}(t)\right) \in \mathrm{PC}_{\frac{3}{2}}[1, e] \times \mathrm{PC}_{\frac{5}{4}}[1, e]$.

Case 2 Take $e(t, u, v)=g(t, u, v)=f(t, u, v)=h(t, u, v)=\sin \sqrt[2]{3} t+e^{-(u+v)^{2}}+\arctan (t u v)$, $I_{1}(u)=\arccos u^{2}, J_{1}(v)=\frac{1}{1+v^{2}}$. Obviously, $e, g, f, h \in C[1, e], I_{1}, J_{1} \in C(\mathbb{R}, \mathbb{R}) .|e(t, u, v)|=$ $|g(t, u, v)|=|f(t, u, v)|=|h(t, u, v)|=\left|\sin \sqrt{3} t+e^{-(u+v)^{2}}+\arctan (t u v)\right| \leq \frac{\pi}{2}+1+\frac{\pi}{2}=\pi+1$, $\left|I_{1}(u)\right|=\left|\arccos u^{2}\right| \leq \pi,\left|J_{1}(v)\right|=\left|\frac{1}{1+v^{2}}\right| \leq 1$. Thus, conditions $\left(H_{1}\right)$ and $\left(H_{4}\right)$ hold. According to Theorem 3.2, we know that (4.1) has at least a pair of solutions $\left(u^{*}(t), v^{*}(t)\right)$.

\section{Conclusions}

In describing some phenomena and processes of many fields such as physics, chemistry, aerodynamics, electrodynamics of a complex medium, polymer rheology, capacitor theory, electrical circuits, biology, control theory, fitting of experimental data, and so on, the fractional differential equation is better and more accurate than the integer-order differential equations. Therefore, the study of fractional differential equations has attracted the eyes of many scholars. Good papers involving the dynamics of the fractional differential equation are emerging in large numbers. However, it was noticed that most of these works are based on Riemann-Liouville and Caputo fractional derivatives. In fact, another kind of fractional derivatives was introduced by Hadamard in 1892. It differs from the aforementioned derivatives in the sense that the kernel of the integral in the definition contains a logarithmic function of arbitrary exponent. Relatively speaking, this fractional differential equation with Hadamard derivatives is still studied less than that of Riemann-Liouville and Caputo. So it is worth studying the Hadamard fractional differential equations. In this paper, we consider the boundary value problem for a class of fractional integro-differential 
coupled systems with Hadamard fractional calculus and impulses. By means of the Banach contraction principle and Leray-Schauder alternative theorem, some new sufficient criteria are established to guarantee the existence and uniqueness of solutions.

\section{Acknowledgements}

The authors thank the referees for a number of suggestions which have improved many aspects of this article.

\section{Funding}

This work was supported by the National Natural Sciences Foundation of Peoples Republic of China under Grant (Nos. 11161025, 11661047).

\section{Availability of data and materials}

Not applicable.

\section{Competing interests}

The authors declare to have no competing interests.

\section{Authors' contributions}

The authors read and approved the final manuscript.

\section{Author details}

${ }^{1}$ Department of Applied Mathematics, Kunming University of Science and Technology, Kunming, China. ${ }^{2}$ School of Mathematics and Computer Science, Panzhihua University, Panzhihua, China.

\section{Publisher's Note}

Springer Nature remains neutral with regard to jurisdictional claims in published maps and institutional affiliations.

Received: 15 January 2019 Accepted: 31 May 2019 Published online: 10 June 2019

\section{References}

1. Ross, B.: The development of fractional calculus 1695-1900. Hist. Math. 4, 75-89 (1977)

2. Miller, K., Ross, B.: An Introduction to the Fractional Calculus and Differential Equations. Wiley, New York (1993)

3. Oldham, K., Spanier, J.: The Fractional Calculus. Academic, New York (1974)

4. Olmstead, W., Handelsman, R.: Diffusion in a semi-infinite region with nonlinear surface dissipation. SIAM Rev. 18, 275-291 (1976)

5. Caputo, M.: Linear models of dissipation whose Q is almost frequency independent - II. Geophys. J. R. Astron. Soc. 13, 529-539 (1967) reprinted in Fract. Calc. Appl. Anal. 11, 4-14 (2008)

6. Caputo, M., Mainardi, F.: A new dissipation model based on memory mechanism. Pure Appl. Geophys. 91, 134-147 (1971) reprinted in Fract. Calc. Appl. Anal. 10, 310-323 (2007)

7. Caputo, M., Mainardi, F.: Linear models of dissipation in anelastic solids. Riv. Nuovo Cimento 1, 161-198 (1971)

8. Marks, I.I., Hall, M.: Differintegral interpolation from a bandlimited signal's samples. IEEE Trans. Acoust. Speech Signal Process. 29, 872-877 (1981)

9. Torvik, P., Bagley, R.: On the appearance of the fractional derivative in the behavior of real materials. J. Appl. Mech. 51, 294-298 (1984)

10. Bai, J., Feng, X.: Fractional-order anisotropic diffusion for image denoising. IEEE Trans. Image Process. 16, 2492-2502 (2007)

11. Chern, J.: Finite element modeling of viscoelastic materials on the theory of fractional calculus. Ph.D. thesis, Pennsylvania State University (1993)

12. Freed, A., Diethelm, K., Luchko, Y.: Fractional-order viscoelasticity (FOV): constitutive development using the fractional calculus (first annual report). Technical Memorandum 2002-211914, NASA Glenn Research Center, Cleveland (2002)

13. Dokoumetzidis, A., Magin, R., Macheras, P.: A commentary on fractionalization of multicompartmental models. J. Pharmacokinet. Pharmacodyn. 37, 203-207 (2010)

14. Popović, J., Atanacković, M., Pilipović, A., Rapaić, M., Pilipović, S., Atanacković, T.: A new approach to the compartmental analysis in pharmacokinetics: fractional time evolution of diclofenac. J. Pharmacokinet. Pharmacodyn. 37, 119-134 (2010)

15. Verotta, D.: Fractional compartmental models and multi-term Mittag-Leffler response functions. J. Pharmacokinet. Pharmacodyn. 37, 209-215 (2010)

16. Freed, A., Diethelm, K.: Fractional calculus in biomechanics: a 3D viscoelastic model using regularized fractional-derivative kernels with application to the human calcaneal fat pad. Biomech. Model. Mechanobiol. 5 203-215 (2006)

17. Magin, R.: Fractional Calculus in Bioengineering. Begell House, Redding (2006)

18. Caponetto, R., Dongola, G., Fortuna, L., Petráš, I.: Fractional Order Systems: Modeling and Control Applications. World Scientific, River Edge (2010)

19. Podlubny, I.: Fractional-order systems and fractional-order controllers. Technical report UEF-03-94, Institute for Experimental Physics, Slovak Acad. Sci. (1994)

20. Kiryakova, $\vee .:$ The special functions of fractional calculus as generalized fractional calculus operators of some basic functions. Comput. Math. Appl. 59, 1128-1141 (2010)

21. Podlubny, I.: Fractional Differential Equation. Academic Press, San Diego (1999) 
22. Kilbas, A., Srivastava, H., Trujillo, J.: Theory and Applications of Fractional Differential. Equations: North-Holland Mathematics Studies., vol. 204. Elsevier, Amsterdam (2006)

23. Samko, S., Kilbas, A., Marichev, O.: Fractional Integrals and Derivatives: Theory and Applications. Gordon \& Breach, Yverdon (1993)

24. Tarasov, V.: Fractional Dynamics: Application of Fractional Calculus to Dynamics of Particles, Fields and Media. Springer, Berlin (2010)

25. Diethelm, K.: The Analysis of Fractional Differential Equations. Springer, Berlin (2010)

26. Goodrich, C.: Existence of a positive solution to a class of fractional differential equations. Appl. Math. Lett. 23, 1050-1055 (2010)

27. Lakshmikantham, V., Leela, S.: Nagumo-type uniqueness result for fractional differential equations. Nonlinear Anal. 71, 2886-2889 (2009)

28. Zhang, X., Liu, L., Wu, Y.: Multiple positive solutions of a singular fractional differential equation with negatively perturbed term. Math. Comput. Model. 55(3), 1263-1274 (2012)

29. Tariboon, J., Ntouyas, S., Sudsutad, W.: Fractional integral problems for fractional differential equations via Caputo derivative. Adv. Differ. Equ. 2014, 181 (2014)

30. Cabada, A., Wang, G.: Positive solutions of nonlinear fractional differential equations with integral boundary value conditions. J. Math. Anal. Appl. 389, 403-411 (2012)

31. Zhao, K., Gong, P.: Existence of positive solutions for a class of higher-order Caputo fractional differential equation. Qual. Theory Dyn. Syst. 14(1), 157-171 (2015)

32. Zhu, C., Zhang, X., Wu, Z.: Solvability for a coupled system of fractional differential equations with integral boundary conditions. Taiwan. J. Math. 17(6), 2039-2054 (2013)

33. Zhao, K.: Multiple positive solutions of integral BVPs for high-order nonlinear fractional differential equations with impulses and distributed delays. Dyn. Syst. 30(2), 208-223 (2015)

34. Yang, W.: Positive solutions for a coupled system of nonlinear fractional differential equations with integral boundary conditions. Comput. Math. Appl. 63(1), 288-297 (2012)

35. Zhao, K.: Impulsive boundary value problems for two classes of fractional differential equation with two different Caputo fractional derivatives. Mediterr. J. Math. 13, 1033-1050 (2016)

36. Henderson, J., Luca, R.: Positive solutions for a system of fractional differential equations with coupled integral boundary conditions. Appl. Math. Comput. 249, 182-197 (2014)

37. Liu, B., Li, J., Liu, L., Wang, Y.: Existence and uniqueness of nontrivial solutions to a system of fractional differential equations with Riemann-Stieltjes integral conditions. Adv. Differ. Equ. 2018, 306 (2018)

38. Agarwal, P., Chand, M., Baleanu, D., O'Regan, D., Jain, S.: On the solutions of certain fractional kinetic equations involving k-Mittag-Leffler function. Adv. Differ. Equ. 2018, 249 (2018)

39. Lu, Z., Zhu, Y.: Comparison principles for fractional differential equations with the Caputo derivatives. Adv. Differ. Equ. 2018, $237(2018)$

40. Xu, M., Han, Z.: Positive solutions for integral boundary value problem of two-term fractional differential equations. Bound. Value Probl. 2018, 100 (2018)

41. Zhang, W., Liu, W.: Existence of solutions for fractional differential equations with infinite point boundary conditions at resonance. Bound. Value Probl. 2018, 36 (2018)

42. Gaafar, F.: The existence of solutions for a nonlinear first-order differential equation involving the Riemann-Liouville fractional-order and nonlocal condition. Mediterr. J. Math. 15, 191 (2018)

43. Zhai, C., Li, P.: Nonnegative solutions of initial value problems for Langevin equations involving two fractional orders. Mediterr. J. Math. 15, 164 (2018)

44. Yukunthorn, W., Suantai, S., Ntouyas, S., Tariboon, J.: Boundary value problems for impulsive multi-order Hadamard fractional differential equations. Bound. Value Probl. 2015, 148 (2015)

45. Benchohra, M., Bouriah, S., Graef, J.: Boundary value problems for nonlinear implicit Caputo-Hadamard-type fractional differential equations with impulses. Mediterr. J. Math. 14, 206 (2017)

46. Ntouyas, S., Tariboon, J., Sudsutad, W.: Boundary value problems for Riemann-Liouville fractional differential inclusions with nonlocal Hadamard fractional integral conditions. Mediterr. J. Math. 13, 939-954 (2016)

47. Ahmad, B., Nitouyas, S.: Boundary value problems of Hadamard-type fractional differential equations and inclusions with nonlocal conditions. Vietnam J. Math. 45, 409-423 (2017)

48. Zhang, Y., Wang, J.: Existence and finite-time stability results for impulsive fractional differential equations with maxima. J. Appl. Math. Comput. 51, 67-79 (2016)

49. Mohamed, I.: On the Hadamard and Riemann-Liouville fractional neutral functional integrodifferential equations with finite delay. J. Pseudo-Differ. Oper. Appl. (2018). https://doi.org/10.1007/s11868-018-0244-1

50. Wang, H., Liu, Y., Zhu, H.: Existence and stability for Hadamard p-type fractional functional differential equations. J. Appl. Math. Comput. 55, 549-562 (2017)

51. Kiataramkul, C., Ntouyas, S., Tariboon, J., Kijjathanakon, A.: Generalized Sturm-Liouville and Langevin equations via Hadamard fractional derivatives with anti-periodic boundary conditions. Bound. Value Probl. 2016, 217 (2016)

52. Ahmad, B., Ntouyas, S., Alsaedi, A.: New results for boundary value problems of Hadamard-type fractional differential inclusions and integral boundary conditions. Bound. Value Probl. 2013, 275 (2013)

53. Alsaedi, A., Ntouyas, S., Ahmad, B., Hobiny, A.: Nonlinear Hadamard fractional differential equations with Hadamard type nonlocal non-conserved conditions. Adv. Differ. Equ. 2015, 285 (2015)

54. Tariboon, J., Ntouyas, S., Sudsutad, W.: Nonlocal Hadamard fractional integral conditions for nonlinear Riemann-Liouville fractional differential equations. Bound. Value Probl. 2014, 253 (2014)

55. Ahmad, B., Ntouyas, S.: On Hadamard fractional integro-differential boundary value problems. J. Appl. Comput. 47, 119-131 (2015)

56. Wang, J., Zhang, Y.: On the concept and existence of solutions for fractional impulsive systems with Hadamard derivatives. Appl. Math. Lett. 39, 85-90 (2015)

57. Huang, H., Liu, W.: Positive solutions for a class of nonlinear Hadamard fractional differential equations with a parameter. Adv. Differ. Equ. 2018, 96 (2018)

58. Thiramanus, P., Ntouyas, S., Tariboon, J.: Positive solutions for Hadamard fractional differential equations on infinite domain. Adv. Differ. Equ. 2016, 83 (2016) 
59. Yang, W.: Positive solutions for singular Hadamard fractional differential system with four-point coupled boundary conditions. J. Appl. Math. Comput. 49, 357-381 (2015)

60. Sudsutad, W., Ntouyas, S., Tariboon, J.: Systems of fractional Langevin equations of Riemann-Liouville and Hadamard types. Adv. Differ. Equ. 2015, 235 (2015)

61. Zhang, X., Shu, T., Cao, H., Ding, W.: The general solution for impulsive differential equations with Hadamard fractional derivative of order $q \in(1,2)$. Adv. Differ. Equ. 2016, 14 (2016)

62. Zhang, X.: The general solution of differential equations with Caputo-Hadamard fractional derivatives and impulsive effect. Adv. Differ. Equ. 2015, 215 (2015)

63. Zhang, W., Liu, W.: Existence of solutions for several higher-order Hadamard-type fractional differential equations with integral boundary conditions on infinite interval. Bound. Value Probl. 2018, 134 (2018)

64. Butzer, P., Kilbas, A., Trujillo, J.: Compositions of Hadamard-type fractional integration operators and the semigroup property. J. Math. Anal. Appl. 269, 387-400 (2002)

65. Butzer, P., Kilbas, A., Trujillo, J.: Fractional calculus in the Mellin setting and Hadamard-type fractional integrals. J. Math. Anal. Appl. 269, 1-27 (2002)

66. Wang, J., Fečkan, M., Zhou, Y.: A survey on impulsive fractional differential equations. Fract. Calc. Appl. Anal. 19(4), 806-831 (2016)

67. Yang, D., Wang, J., O'Regan, D.: On the orbital Hausdorff dependence of differential equations with non-instantaneous impulses. C. R. Math. 356(2), 150-171 (2018)

68. Guo, D., Lakshmikantham, V.: Nonlinear Problems in Abstract Cone. Academic Press, Orlando (1988)

\section{Submit your manuscript to a SpringerOpen ${ }^{\circ}$ journal and benefit from:}

- Convenient online submission

- Rigorous peer review

- Open access: articles freely available online

- High visibility within the field

- Retaining the copyright to your article

Submit your next manuscript at $\gg$ springeropen.com 